\title{
Chondrogenic Commitment of human Bone Marrow Mesenchymal Stem Cells cultured under perfusion within a 3D collagen environment releasing hTGF $\beta 1$
}

\author{
Erwin Lamparelli ${ }^{1}$, Joseph Lovecchio ${ }^{2}$, Luigi Marino ${ }^{1}$, Maria Ciardulli ${ }^{1}$, Carmine Selleri $^{1}$, \\ Nicholas Forsyth $^{3}$, Emanuele Giordano ${ }^{4}$, Nicola Maffulli ${ }^{5}$, and Giovanna Della Porta ${ }^{1}$ \\ ${ }^{1}$ University of Salerno - Baronissi Campus \\ ${ }^{2}$ University of Bologna \\ ${ }^{3}$ Keele University \\ ${ }^{4}$ Università di Bologna \\ ${ }^{5}$ University of Salerno
}

June 24, 2020

\begin{abstract}
The optimal growth, maturation and function of bioengineered tissues are mediated by both biochemical and physical cues. We here describe a 3D biomimetic environment directing stem cells towards a chondrogenic phenotype. This system comprises a collagen hydrogel and poly-lactic-co-glycolic acid microcarriers (PLGA-MCs) engineered to protect, carry and release a human Transforming Growth Factor b1 (hTFGb1) payload. PLGA-MCs were prepared using supercritical emulsion extraction technology and integrated into a collagen hydrogel co-seeded with human Bone Marrow Mesenchymal Stem Cells (hBM-MSCs). Testing different concentrations of hTFGb1 supplemented to cell monolayer cultures suggested $10 \mathrm{ng} / \mathrm{mL}$ as the most appropriate concentration to promote upregulation of SRY-Related HMG-BOXGene 9 (4-fold) and collagen type II (2-fold) specific markers, at Day 16. A similar growth factor concentration was delivered within the 3D bioengineered environment cultured in a dynamic via a custom perfusion bioreactor. A chondrogenic commitment was obtained as indicated by upregulation of collagen type II (5-fold) and downregulation of collagen types I and III (both 0.1-fold) at Day 16. Histological analysis confirmed the remodeling of the synthetic extracellular matrix in where an enhanced mass exchange was described by FEM analysis of fluid-dynamics and related nutrient mass transfer within the 3D construct. This study supports the use of 3D bioengineered scaffolds cultured in a dynamic environment as a suitable tissue engineered model to study chondrogenic differentiation in vitro and opens perspectives for an injectable collagen-based advanced therapy system.
\end{abstract}

\section{INTRODUCTION}

Osteoarthritis (OA) is caused by progressive wear of joints (Faust et al., 2019), and is often a consequence of articular cartilage injuries. The incidence of OA increases with age, and it is one of the most prevalent diseases in elderly people (Zhang et al., 2016; Zhang and Jordan, 2010). The avascular nature of cartilage prevents spontaneous healing; moreover, conventional pharmacological therapy is directed toward symptomatic treatment, mainly aimed at pain reduction, and is not able to promote regeneration of impaired cartilage (Harrell et al., 2019). Patients who do not respond to pharmacological therapies ultimately undergo surgical procedures to promote cartilage repair, such as arthroscopic debridement, bone marrow stimulation techniques, autologous chondrocyte implantation, and, finally, total or partial joint replacement (Rönn et al., 2011). In the bone marrow stimulation technique, sub-chondral drilling induces bleeding, which enables mesenchymal cells from the bone marrow to migrate into the site of injury. The repaired tissue is primarily 
composed by fibrocartilage, with poorer biochemical and biomechanical characteristics than hyaline cartilage. Consequently, clinical outcomes are not always adequate (Pastides et al., 2013).

Tissue engineering (TE) protocols offer great potential to improve joint cartilage therapy (Mollon et al., 2013). TE involves the use of 3D scaffolds made of biocompatible and biodegradable polymers, able to host stem cells (SCs) and growth factors to promote their differentiation towards a chondrogenic phenotype. The mechanical and biochemical properties of scaffolds should reproduce the complex architecture of structural proteins arranged in specific three-dimensional design of natural extracellular matrix (ECM) (Badylak et al., 2008). The ECM of articular cartilage is mainly composed by hydrated collagen (mainly, type II) and proteoglycans (such as aggrecan), with high content of retained water to ensure and maintain its specific mechanical properties (Sophia Fox et al., 2009).

TE scaffolds are manufactured with biodegradable and biocompatible materials, which may be natural or synthetic. The choice of scaffold is crucial to the success of the therapeutic strategy (Cipollaro et al., 2019). Collagen-based scaffolds seem more appropriate to host chondrocytes, being more similar to natural ECM (El-Sherbiny and Yacoub, 2013; Madry et al., 2014). Particularly, collagen hydrogels (type I) are the most investigated as they are suitable for implantation given their biocompatibility in vivo, and the main sources of collagen include bovine skin and rat tail (Parenteau-Bareil et al., 2010).

Growth factors such as TGF $\beta 1$ and TGF 33 have chondrogenic potential and are often used with SCs. TGF $\beta 1$ is the most widely investigated growth factor to induce chondrogenesis in $h$ MSCs and human adipose stem cells ( $h$ ASCs), even though its optimum concentration to be supplemented is under debate. For example, Yoo et al. (1998) reported that $h$ BM-MSCs cultured with a TGF $\beta 1$ concentration ranging from 0.01 to 0.1 $\mathrm{ng} / \mathrm{mL}$ acquired a polygonal morphology, but, at higher concentrations $(0.5$ or $1.0 \mathrm{ng} / \mathrm{mL})$ the cells became spindle-shaped and detached from the culture dishes in absence of chondrogenic differentiation (Puetzer et al., 2010; Yoo et al., 1998). In other studies, $10 \mathrm{ng} / \mathrm{mL}$ TGF $\beta 1$ induced hACS nodule formation and sulfated proteoglycan and aggrecan expression, while $100 \mathrm{ng} / \mathrm{mL}$ reduced this chondrogenic potential (Huang et al., 2004). Growth factors are mainly administered using a supplemented medium which has to be replaced frequently, with a high economic impact due to the relevant cost of recombinant peptides.

Recently, the encapsulation of growth factors into biopolymeric micro/nano carriers was suggested to promote their controlled local release within 3D microenvironment for tissue engineering application (Della Porta et al., 2015; Della Porta et al., 2018; Ciardulli et al., 2020). Micro/nano carriers can be fabricated with several technologies based on the emulsions processing by evaporation/extraction of the oily phase and collecting the resulting biopolymer beads. Dense gas technology has been described for oily phase extraction, allowing an accurate carrier size control and good growth factor or drug loading (Della Porta et al., 2017; Tirado et al., 2019; Palazzo et al., 2020; Govoni et al., 2020). These carriers can be easily immobilized within the hydrogel matrix, and the controlled delivery of their cargo within the 3D scaffold can be accomplished. As a consequence, it is possible to optimize the cells/carrier ratio to maintain a constant growth factor concentration within the 3D system (Trucillo et al., 2019).

In our experience, dynamic conditions are a significant improvement for 3D culture systems (Lovecchio et al. 2019 and 2020; Govoni et al, 2014, 2016 and 2017; Ciardulli el al, 2020). Perfusion appears to upregulate chondrogenic markers (Alves da Silva et al., 2011; Tiğlı et al., 2011), preserve the chondrogenic phenotype and to prevent hypertrophy of MSC-derived chondrocytes, which remains an issue in cell-based strategies (Fuentes-Mera et al., 2017).

Following these considerations, the present study aimed at assembly a collagen 3D scaffold loaded with human MSC derived from Bone Marrow (hBM-MSCs) and poly-lactic-co-glycolic acid microcarriers (PLGA-MCs) with hTGF $\beta 1$ payload to study their chondrogenic commitment. First, to check the amount of relevant growth factor to be delivered within the 3D system, the effect of hTGF $\beta 1$ supplemented at different concentrations was monitored following the gene expression of collagen type II, SRY-Related HMG-BOXGene 9, aggrecan (positive markers) and collagen type I and III (negative ones) by hBM-MSCs in monolayer culture. Quantitative immunofluorescence ( $\mathrm{q}-\mathrm{IF}$ ) was also used to detected collagen II and III proteins. 
PLGA-MCs were fabricated by dense gas proprietary technology, assayed for size, loading and release profiles and assembled into 3D bioengineered system cultured in a custom perfusion bioreactor. FEM analysis to assess the nutrient consumption and waste production within the 3D scaffold was performed and RT-qPCR, immunohistology and q-IF were used to describe the culture outcomes.

\section{MATERIALS AND METHODS}

\section{hBM-MSCs isolation and harvesting}

$h$ BM-MSCs were obtained from the bone marrow of independent healthy donors (age between 40 and 38). The donors gave written informed consent in accordance with the Declaration of Helsinki to the use of their filter residual bone marrow aspirate for research purposes, with approval from the University Hospital of San Giovanni di Dio e Ruggi d'Aragona (Salerno, IT). Review Board (prot./SCCE n. 24988 achieved on April 09th, 2015).

Briefly, total bone marrow aspirate was directly seeded at a concentration of 50,000 total nucleated cells $/ \mathrm{cm}^{2}$ in T75 plastic flask in Minimum Essential Medium Alpha ( $\alpha$-MEM) supplemented with $1 \%$ Glutagro $^{\text {TM }}, 10 \%$ Fetal Bovine Serum (FBS) and $1 \%$ Pen/Strep and incubated at $37^{\circ} \mathrm{C}$ in $5 \% \mathrm{CO}_{2}$ atmosphere and $95 \%$ relative humidity (Giordano et al., 2014). After 72h, non-adherent cells were removed by medium change and the adherent cells were further fed twice a week with new medium. On day 14, colonies of adherent $h$ BM-MSCs were detached and re-seeded at 4000 cells $/ \mathrm{cm}^{2}$ in the same culture conditions. Once the cell cultures reached $70-80 \%$ confluence (7-8 days for the previous passage), cells were detached using $0.05 \%$ trypsin- $0.53 \mathrm{mM}$ EDTA and were washed with PBS 1X (Corning Cellgro, Manassas, VA, USA), counted using Trypan Blue (Sigma-Aldrich, Milan, IT) and subcultured at a concentration of $4 \times 10^{3}$ cells $/ \mathrm{cm}^{2}$. At Passage 2, cells were used for the experiments. Flow cytometry analysis was performed on $h$ BM-MSCs obtained at Passage 2; cells were positive for CD90, CD105, CD73 and negative for CD14, CD34, CD45, HLA-DR expression (Miltenyi Biotec, DE).

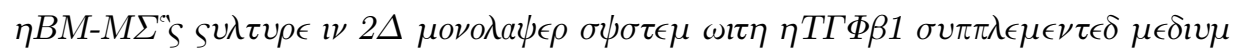

$h$ BM-MSCs (passage 2) were seeded on coverslips in 12 well-plates at a concentration of $4 \times 10^{3} \mathrm{cells} / \mathrm{cm}^{2}$. Once the cell cultures reached $60 \%$ confluence, cells were treated with $1 \mathrm{ng} / \mathrm{mL}$ or $10 \mathrm{ng} / \mathrm{mL}$ of recombinant human TGFß1 (PeproTech; UK). Cells were fed twice a week with new medium and a new dose of growth factor for up to 16 days. Untreated cells for all the time-points studied were used as control.

\section{PLGA-MCs fabrication by Supercritical Emulsion Extraction technology}

Poly-Lactic-co-Glycolic Acid micro-carriers (PLGA-MCs) were obtained using Supercritical Emulsion Extraction (SEE) technology via dense gas extraction of emulsion oily phase. The process layout is described by a countercurrent packed tower operating in continuous mode (Della Porta et al., 2011; Gimenez-Rota et al., 2019). PLGA-MCs were fabricated using awater-oil-water emulsion (ratio 0.25:5:50). In detail, recombinant hTGFß1 (PeproTech; UK) was dissolved into 0.1\% w/v human serum albumin (hSA; Sigma-Aldrich, Milan, IT) containing $0.06 \% \mathrm{w} / \mathrm{w}$ of polyvinyl alcohol (PVA; Sigma-Aldrich, Milan, IT) used as surfactant; hSA was included as growth factor stabilizer into primary emulsion (Nihant et al., 1995). This water phase was added to the oily phase formed in ethyl acetate (EA, purity 99.9\%) and PLGA (RESOMER ${ }^{\circledR}$ RG 504H, molecular weight: $38000-54000 \mathrm{kDa}$ from EVONIK) at $5 \% \mathrm{w} / \mathrm{w}$. The water/oil $(w / o)$ emulsification was produced by vortex mixer for $30 \mathrm{~s}$ at maximum speed; the resulting primary emulsion was immediately poured into EA-saturated aqueous solution containing $0.1 \% \mathrm{w} / \mathrm{w}$ Tween 80 and $15 \% \mathrm{w} / \mathrm{w}$ glucose, before the secondary emulsion was formed via a high-speed homogenizer (mod. L4RT; Silverson Machines Ltd., Waterside, Chesham Bucks, UK) at $2000 \mathrm{rpm}$ for $5 \mathrm{~min}$ and at $10^{\circ} \mathrm{C}$. All emulsions were processed by SEE immediately after their preparation.

Operative pressure and temperature conditions in the high-pressure column were set at $8 \mathrm{MPa}$ and $38^{\circ} \mathrm{C}$, respectively, with a dense gas flow of Carbon Dioxide $\left(\mathrm{CO}_{2}\right)$ set at $1.4 \mathrm{~kg} / \mathrm{h}$ with Liquid/Gas ratio of $0.1 \mathrm{w} / \mathrm{w}$ (Della Porta et al., 2013). PLGA-MCs suspensions were collected at the bottom of the extraction column, washed to eliminate surfactant, and lyophilized. 
To ensure a cell culture-grade preparation, the washing steps were performed in sterile condition with a pen/strep and amphotericin B (1\% w/v) solution.

Carriers size distribution and morphological analyses

Particle size distributions (PSDs) of PLGA-MCs suspension were measured using a laser granulometer (mod. Mastersizer S; Malvern Instruments Ltd., Worcestershire, UK), based on dynamic light scattering (DLS). Sizes are expressed as volume mean size (MS) with standard deviation (SD) in nanometers (nm). The shape and morphology of the PLGA-MCs were investigated by field emission-scanning electron microscopy (FESEM, mod. LEO 1525; Carl Zeiss, Oberkochen, D). PLGA-MCs samples were placed on a double-sided adhesive carbon tape previously glued to an aluminum stub and coated with a gold film (250 A thickness) using a sputter coater (mod.108 A; Agar Scientific, Stansted, UK).

\section{$\eta T \Gamma \Phi \beta 1 \rho \epsilon \lambda \epsilon a \sigma \epsilon \sigma \tau \tau \delta \psi$}

The release profile of hTGF $\beta 1$ from PLGA-MCs was monitored in vitro by using $5 \pm 0.3 \mathrm{mg}$ of PLGA-MCs suspended in $500 \mu \mathrm{L}$ of PBS $1 \mathrm{X}$ containing $0.1 \%$ Tween 20 and $0.1 \% \mathrm{hSA}$, placed in an incubator at $37^{\circ} \mathrm{C}$, and stirred continuously at $100 \mathrm{rpm}$. Every 24 hours, the samples were centrifuged at $14000 \mathrm{rpm}$ for $30 \mathrm{~min}$, and the supernatant completely removed and replaced with fresh PBS to maintain sink conditions. Released hTGFß1 concentrations from collected samples were then measured with an Enzyme Linked Immunosorbent Assay (ELISA, Cloud-Clone Corp., USA). Release experiments were performed in triplicate $(\mathrm{n}=3)$, and the curve describing the mean profile calculated as $\mathrm{ng} / \mathrm{g}$ (protein released/PLGA-MCs) versus time or in percentage amount calculated with respect the maximum growth factor load.

\section{$3 D$ collagen scaffold preparation and characterization}

Cylindrical 3D scaffolds were prepared by gelling collagen with $h$ BM-MSCs and PLGA-MCs loaded with hTGF $\beta 1$. For each scaffold, a mixture of collagen type I from rat tail (Advanced BioMatrix; US), neutralization solution (Advanced BioMatrix; US), and $\alpha$-MEM (Corning, NY, USA) supplemented with $10 \%$ fetal bovine serum (referred to as growing medium, GM) was added at a 9:1:2 ratio. The $\alpha$-MEM contained an overall amount of $6.6 \mathrm{mg}$ of PLGA-MCs (7 ug/g hTGF $\beta 1 / P L G A)$ and an average of $2.2 \times 10^{5}$ cells. This suspension was pipetted in different wells of a 96 -well plate then placed into the incubator at $37^{\circ} \mathrm{C}$ for 60 min to allow collagen thermal cross-linking. 3D scaffolds were then transferred to a culture plate.

The viability of cells into scaffolds was detected by fluorescence live/dead assay, just after their preparation. Cells were stained for 1 hour at $37^{\circ} \mathrm{C}$, washed in PBS 1X and imaged in a fluorescence microscope (mod. Eclipse, Nikon, DE). Green emission of the Calcein dye stains the cytosol of live cells; while red emission of cell membrane-impermeable ethidium homodimer-1 dye stains nuclei of dead cells.

The internal morphology of the scaffold was observed by field emission-scanning electron microscopy (FESEM, mod. LEO 1525; Carl Zeiss, Oberkochen, DE). Samples were fixed in $4 \%$ PFA $\left(4{ }^{\circ} \mathrm{C}\right.$, overnight) and then dehydrated by multiple passages across ethanol:water solutions (10 minutes each) with increasing percentages of ethanol $(10 \%, 20 \%, 30 \%, 50 \%, 70 \%, 90 \%)$, ending in a $100 \%$ dehydrating liquid (3 changes, 10 minutes each), and lyophilized using a critical point dryer (mod. K850 Emitech, Assing, Rome IT). Samples were immersed in liquid nitrogen and fractured with a needle, then placed on a double-sided adhesive carbon tape previously glued to an aluminum stub and coated with a gold film (250 A thickness) using a sputter coater (mod.108 A; Agar Scientific, Stansted, UK), before observation.

\section{Dynamic culture}

3D scaffolds were placed in a perfusion bioreactor, formed by a custom multiwell plate was milled in poly(methyl methacrylate) (PMMA, Altuglas®) CN 100 10000, Altuglas International, La Garenne-Colombes Cedex, FR), a biocompatible material for biomedical applications (Samavedi et al., 2014). This plate has two holes which allow the insertion of silicon tubes (Tygonß, FR) for the medium flow provided by peristaltic pumps at constant flow rate of $1.0 \mathrm{~mL} / \mathrm{min}$ (Pasini et al., 2019). The bioreactor system operated within a standard cell culture incubator. 


\section{Immunofluorescence assay}

At different time points, cells were fixed with $3.7 \%$ formaldehyde for $30 \mathrm{~min}$ at room temperature (RT) followed by permeabilization with $0.1 \%$ Triton X-100 for 5 min and blocked with BSA solution (1\% w/v) for $1 \mathrm{~h}$. For type II and III collagen staining, cells were incubated overnight at $4 \mathrm{deg} \mathrm{C}$ with a rabbit polyclonal anti-collagen type II antibody (1:100, Abcam, UK) and mouse polyclonal anti-collagen type III antibody (1:100, Santa Cruz Biotech., USA). Following incubation with the primary antibody, cells were incubated for $1 \mathrm{~h}$ at RT with the Alexa Fluor ${ }^{\mathrm{TM}} 488$ goat anti-rabbit IgG (1:400, Thermo Fisher Scientific, USA) and the DyLight 649 anti-mouse IgG (1:500, BioLegend, CA. USA) antibody. Cell nuclei were counterstained with DAPI.

At different time points, a portion of each collagen scaffold was fixed in $4 \%$ PFA for $2 \mathrm{~h}$ at room temperature, cryo-protected in 30\% sucrose (4degC, overnight), included in OCT, and cut in slices of $15 \mu \mathrm{m}$ of thickness using a cryostat (mod. CM1950, Leica, Wetzlar, Germany). For type II and type III collagen staining, slices were incubated overnight at $4^{\circ} \mathrm{C}$ with a rabbit polyclonal anti-collagen type II antibody (1:100, Abcam, UK) and mouse polyclonal anti-collagen type III antibody (1:100; Santa Cruz Biotech., USA). Following incubation with the primary antibody, slices were incubated for $1 \mathrm{~h}$ at RT with the Alexa Fluor ${ }^{\mathrm{TM}} 488$ goat anti-rabbit IgG (1:400, Thermo Fisher Sci., USA) and the DyLight 649 anti-mouse IgG (1:500, BioLegend, CA, USA) antibodies.

Single images were acquired with the same settings of light intensity, exposure time and gain using an inverted Leica laser-scanning confocal microscope (mod. TCS SP5; Leica Microsystems, Wetzlar DE) equipped with a plan Apo 63X/1.4 NA oil immersion objective. Signals intensity, related to the proteins of interest, was quantified using the software ImageJ (rel.1.52p National Institutes of Health, USA). At first, the original images in RGB format were converted into a 16-bit (gray scale) format. Thereafter, the tagged areas were expressed as an average value of pixels intensity (within a range from 0 (dark) to 255 (white). These data were normalized considering the number of cells present in the whole field (Suchorska et al., 2016). Five images of several fields were used for the analysis at each time point. All data were reported as fold change relative to untreated cells.

\section{Hematoxylin and eosin and Sirius red staining}

Scaffold slices (15 $\mu \mathrm{m}$ thickness) were hydrated using decreasing ethanol gradient to $75 \%$ and washed for 5 min in water. The hydrated slices were nuclear stained using hematoxylin for $5 \mathrm{~min}$, and then counterstained using eosin for $1 \mathrm{~min}$; thereafter, the sections were dehydrated in increasing EtOH gradient and finally cleared in xylene for $5 \mathrm{~min}$.

In the case of Sirius red staining, Picrosirius Red Stain Kit of Polysciences, Inc. was used on the same sample slices stained in hematoxylin for $8 \mathrm{~min}$ and washed in water for $2 \mathrm{~m}$. The sections were dipped into phosphomolybdic acid for $2 \mathrm{~min}$ and washed in water for $2 \mathrm{~min}$. Then they were dipped into Picrosirius Red F3BA Stain for $60 \mathrm{~min}$ and dipped into $\mathrm{HCl} 0.1 \mathrm{M}$ solution for $2 \mathrm{~m}$. The sections were dehydrated in increasing ethanol gradient solutions and finally dipped into xylene for $5 \mathrm{~min}$. Sections were mounted using Eukitt (Sigma-Aldrich, Milan, IT) mounting medium.

\section{$R N A$ isolation and gene expression profile}

mRNA expressions were analyzed by Reverse Transcription quantitative polymerase chain reaction (RTqPCR). Chondrogenic markers such as type II collagen (Col2a1), SRY-Box transcription factor 9 (Sox9), aggrecan (Acan), and negative marker of chondrogenesis such as type I and III collagen (Col1a1 and Col3a1 ) were investigated. Total RNA was extracted from $h$ BM-MSCs seeded into the 12 well-plates and from collagen scaffolds of each time point using QIAzol@ Lysis Reagent (Qiagen, DE), chloroform (Sigma-Aldrich, Milan, IT) and the RNeasy Mini Kit (Qiagen, DE). For each sample, $1 \mu \mathrm{g}$ of total RNA was reverse transcribed using the iScriptTM cDNA synthesis kit (Bio-Rad, Milan, IT). Relative gene expression analysis was performed in a LightCycler@ 480 Instrument (Roche, IT), using the SsoAdvanced ${ }^{\mathrm{TM}}$ Universal SYBR@ Green Supermix (Bio-Rad) with the validated primers for Col1A1, Col2A1, Col3A1 , Sox9 , and 
Acan (Bio-Rad, USA), and following MIQE guidelines (Bustin et al., 2009). Amplification was performed in a $10 \mu \mathrm{L}$ final volume, including $2 \mathrm{ng}$ of complementary DNA (cDNA) as template. The specificity of the formed products was addressed via melting curve analysis. Duplicate experiments were performed for each condition explored, and data were normalized to glyceraldehyde-3-phosphate dehydrogenase (GAPDH ) expression (reference gene), applying the geNorm method (Hellemans et al., 2007) to calculate reference gene stability between the different conditions (calculated with CFX Manager software; $\mathrm{M}<0.5$ ). Fold changes in gene expression were determined by the $2^{-\Delta \Delta^{n} \Pi}$ method and are presented as relative levels versus untreated cells at each time-point explored.

\section{Statistical analysis}

The results are given as the mean \pm standard deviation (SD). Statistical analysis was performed using the two-tailed independent Student's T test for comparisons of two independent groups. To compare the influences of the chemical and dynamic stimulation within the same time point, two-way ANOVA was used followed by Tukey's multiple comparison test. $\mathrm{P}$ values less than 0.05 were accepted as significant (de Winter, 2013). All statistical analysis was conducted using GraphPad Prism software (6.0 for Windows).

\section{FEM modeling}

Finite Element Modeling (FEM) was implemented using COMSOL Multiphysics Software to assess the nutrient consumption and waste production in static versus dynamic conditions during the cell culture. Both the well and the scaffold have a cylindrical geometry (well $=22 \mathrm{~mm}$ in diameter and $7.4 \mathrm{~mm}$ in height; scaffold $5 \mathrm{~mm}$ in diameter and $5 \mathrm{~mm}$ in height). All components were obtained using primitive geometries and Boolean operations. The assumed boundary conditions were laminar flow and mass transport. Hydrogel estimated value for permeability was assumed as $2 * 10^{-9} \mathrm{~cm}^{2}$ (Knapp et al., 1997). A sensitivity study of the mesh addressed the most computationally efficient solution. The boundary conditions, constants and main parameters used in the model were derived from Lovecchio et al. (2019) and Pasini et al. (2019).

\section{RESULT AND DISCUSSION}

$2 \Delta \varsigma v \lambda \tau v \rho \epsilon \sigma \tau v \delta \psi \omega \imath \tau \eta \eta T \Gamma \Phi \beta 1 \sigma v \pi \pi \lambda \epsilon \mu \epsilon \nu \tau \epsilon \delta \mu \epsilon \delta \imath v \mu$

$h$ BM-MSCs commitment towards a chondrogenic phenotype was studied in the presence of TGF $\beta 1$ at different concentrations to estimate how to set the cells/carrier ratio within the $3 \mathrm{D}$ system studied in the present investigation. Therefore, two different doses ( 1 and $10 \mathrm{ng} / \mathrm{mL}$ ) of hTGF $\beta 1$ were administered during 16 days of culture. Untreated cells for all the time-points investigated were used as control. The effect on the expression of positive (collagen type II,Col2a1 ; SRY-Box transcription factor 9, Sox 9 ; and aggrecan, Acan ) and negative (collagen type I and III,Col1a1 and Col3a1) chondrogenic markers by $h$ BM-MSCs is reported in Figure 1a-b.

In the chondrogenic commitment of human MSCs, the transcription factorSox 9 plays a key role and is considered as an early chondrogenic marker; its upregulation drives the correct chondrogenic phenotype, decreasing the hypertrophic drift of the cells (Jiang et al., 2018) which would gradually promote mineralization of hyaline cartilage and result in apoptosis and ossification (Mueller and Tuan, 2008). At day 16, supplementing $1 \mathrm{ng} / \mathrm{mL}$ of hTGF $\beta 1$, Sox9 levels doubled (Figure 1a); whereas, using $10 \mathrm{ng} / \mathrm{mL}$, Sox9 showed a fourfold up-regulation (Figure 1b ).

Col2a1 represents the gold standard chondrogenic marker, which is usually detectable only after several days of chondrogenesis induction. This gene was not overexpressed in all time points observed when using $1 \mathrm{ng} / \mathrm{mL}$; whereas supplementing $10 \mathrm{ng} / \mathrm{mL}$, its expression appeared doubled at Day 16 . These data are in agreement with Li et al. (2011), who reported that collagen type II expression in monolayer culture, after treatment with hTGF $\beta 1$, remained undetectable at earlier time points ( $2 \mathrm{~h}$ to 3 days) and becomes elevated only after 2 weeks (Li et al., 2011).

Acan is the third positive marker, but it was not detected at any time point at both hTGF $\beta 1$ concentrations explored, possibly because it has been indicated to be expressed between 18-24 days of culture (Xu et al., 
2008).

Col1a1 and Col3a1 were checked as negative markers because they are largely reported to be dedifferentiation genes of hyaline cartilage phenotype (Dehne et al., 2010). Indeed, type III collagen is related to a fibrocartilaginous phenotype and is often co-localized with type I collagen within the same fibril (Dehne et al., 2010). Type I collagen, instead, is the main component of fibrocartilage tissue (Liu et al., 2017), and increases in chondrocytes during the progression of human osteoarthritis (Zhong et al., 2016). The exhibition of a fibroblast-like phenotype during cultivation compromises the clinical outcome of potential regenerative therapy, and becomes a major issue for the application of hBM-MSC in the repair of cartilage defects (Li et al., 2019). Effectively, the expression of both these genes was down regulated, better when $10 \mathrm{ng} / \mathrm{mL}$ was added in the culture medium (Figure 1a-b ).

The effect of hTGF $\beta 1$ on the production of COL2A1 and COL3A1 proteins was monitored by quantitative immunofluorescence assay (Figure 2 ). hBM-MSCs showed slight basal expression of both COL2A1 and COL3A1; however, COL2A1 staining (in green) was more intense when cultivated with $10 \mathrm{ng} / \mathrm{mL}$ at day 16; this behavior was confirmed by q-IF data which indicate an $30 \%$ increase of the stained protein signal at day 16. In the same conditions, COL3A1 signal was only slightly detected. Supplementing $1 \mathrm{ng} / \mathrm{mL}$, several spindle shaped system aggregates were observed at day 16 . No spindle systems were evidenced when a concentration of $10 \mathrm{ng} / \mathrm{mL}$ was used. These spindle structures have already been reported by Puetzer et al., who suggested that the chondrogenesis commitment of MSCs is better promoted by $10 \mathrm{ng} / \mathrm{mL}$ hTGF $\beta 1$ (Puetzer et al., 2010); indeed, these structures may be considered an early organization of cellular 3D structure in the context of tenogenic commitment (Barboni et al., 2012) and, in this sense, the concentration of $1 \mathrm{ng} / \mathrm{mL}$ may also play a role in others different differentiation pathways.

It is worth of note that despite several studies suggested to supplement dexamethasone (at about $100 \mathrm{nM}$ ) to the medium to improve the effect of TGF- $\beta 1$ and enhance stem cells differentiation (Shintani and Hunziker, 2011), in our experiments we excluded this option in order to monitor the effect of growth factor alone on cells behavior, and, in the follows the cooperative effect of 3D environment. So following these preliminary data, we adopted a concentration of about $10 \mathrm{ng} / \mathrm{mL}$ as the optimal dose for the following study.

\section{$3 D$ culture assembly and characterization}

PLGA-MCs were fabricated by proprietary technology that uses dense gas to provide a micro sized system for the sustained local delivery of hTGF $\beta 1$ ( Della Porta et al., 2013) . PLGA-MCs displayed a spherical morphology with a mean size of $3 \pm 0.9 \mu \mathrm{m}$ as illustrated inFigure 3 where SEM imaging of the carriers (a) and their particle size distribution (b) are reported. PLGA-MCs are not amenable to conventional sterilization methods such as ethanol washing, gamma irradiation, or steam as these methods would produce biopolymer oxidation, loss of microstructure, size and morphology, and degradation of peptide payload. To overcome these challenges, a specific SEE operational protocol for sterile materials production was used, and PLGA-MCs were recovered in a sterile suspension (Della Porta et al., 2018). PLGA-MCs showed a hTGF $\beta 1$ loading of $7 \mu \mathrm{g} / \mathrm{g}$, and provided a sustained release profile over 35 days into PBS at $37^{\circ} \mathrm{C}$ (see Fig 3c ). A total amount of $6.6 \mathrm{mg}$ of PLGA-MCs was loaded within each 3D collagen scaffold (volume $320 \mu \mathrm{L}$ ) together with 200,000 cells. The estimated hTGF $\beta 1$ release within the scaffold is presented inFigure 3d. A burst release of $20 \mathrm{ng} / \mathrm{mL}$ is observed at day 1 , a mean amount of $4 \mathrm{ng} / \mathrm{mL} /$ day for the following 5 days, and $0.5 \mathrm{ng} / \mathrm{mL} /$ day are delivered in the following days, except at day 13 , when a spike of $5 \mathrm{ng} / \mathrm{mL}$ results from PLGA burst de-polymerization (Trucillo et al., 2019). An overall amount of $45 \mathrm{ng}$ of hTGF $\beta 1$ is thus delivered in each scaffold over the 16 days of culture time (Reyes et al., 2012).

The schematic representation of the 3D scaffold assembly protocol and a scaffold picture (after 16 days of culture) are illustrated inFigure $\mathbf{4 a} \mathbf{a} \mathbf{b}$. To better understand the scaffold architecture and proper cells and PLGA carriers loading and distribution within the 3D structure, PLGA-MCs loaded with rhodamine B (Rod-B, $8 \mathrm{mg} / \mathrm{g}$ ) were used for a preliminary scaffold assembly to observe at confocal microscopy the carrier dispersion within it together with the cells. A confocal microscopy image of the scaffold structure is shown in Figure 4c, where cell dispersion can be observed looking at the blue dots (DAPI for cells nuclei) and 
PLGA-MCs are highlighted as red spots. The image clearly demonstrated a homogeneous distribution of cells and carriers within the scaffold. The collagen 3D scaffold was then fixed, dehydrated and freeze-fractured for FE-SEM images acquisition (Figure 4d ) to observe the collagen fibers network can be observed.

During the mastermix extrusion, the distribution of velocity and shear stress across the cross-section of the pipette nozzle are different, and cells experience shear stresses, particularly at the wall areas (Li et al., 2018). Therefore, since the shear stress is an inevitable factor in any dispensing process, hBM-MSCs viability after collagen thermic cross-linking was studied by Live and Dead protocols (Figure 5a-b). In our case, an excellent cell viability, with $97 \%$ of live cells, was observed.

Microstructural model for collagen gel deformations. This schematic of a collagen gel in shear and compression shows how fibrils are extended in shear while they buckle in confined compression. These differences in microstructural deformation can affect the macroscopic mechanical properties of the gel.

3D constructs were maintained in dynamic culture conditions in a custom perfusion bioreactor (Figure 6a ) to ensure a $1 \mathrm{~mL} / \mathrm{min}$ of medium flow rate through each well. (Figure $\mathbf{6 b}$ ). The FEM analysis of the nutrient concentration within a single culture well, in static vs. dynamic conditions, was performed and is illustrated in Figures 6c,d,f,g . A laminar flow (grey lines) and a uniform velocity distribution (horizontal cross-section) are maintained within every single culture well (Figure 6e). In detail, in static conditions the in silico -model shows (Figure 6c ) an uneven nutrient distribution within the well, with the lower concentration within the $3 \mathrm{D}$ construct. On the other hand, a uniform nutrient distribution is maintained (Figure 6f ), in the whole well medium/scaffold volume in dynamic conditions. As a consequence, 1 order of magnitude more nutrient concentration was estimated within the perfused vs. the static scaffold $\left(10^{-8}\right.$ vs. $10^{-7}$ $\mathrm{mol} / \mathrm{m}^{3}$ ). A mirror image is obtained when the waste products are considered (Figure $6 \mathbf{d}, \mathbf{g}$ ). Our data are in agreement with those reported in the literature where cells immobilized within a 3D system are described under risk of reduced metabolites and oxygen exchanges that may be responsible of impaired metabolic activity in static culture environment (Della Porta et al., 2015; Yeatts and Fisher, 2011). Moreover, the fact that the overall mass exchanges are favored by perfusion also suggest an adequate environment to ensure the appropriate sink conditions which enforce the sustained release of growth factor from PLGA carriers dispersed within the 3D system (Siepmann and Siepmann, 2008). The absence of proper mass exchange would negatively affect the effective release of growth factor because of an external resistance to the mass transfer and justify a poor outcome of static culture of the same assembled scaffold in where all the checked chondrogenic markers were down regulated (data not shown).

$3 D$ culture outcomes

Scaffolds collected at 8 and 16 days were stained with either Sirius Red or Hematoxylin and Eosin (Figure 7) . At day zero, cells can be clearly seen homogeneously dispersed within the gel matrix (seeFigure 7a enlarged with arrowheads); matrix reshaping is observed along time coherently with the progressive deposition new and ordinate matrix by the cells (see inset in Figure 7c ). Sirius staining highlighted the neo-synthesized collagen component within the randomly assembled synthetic matrix (Figure $\mathbf{7 d}$ ). This original scaffold matrix appeared progressively remodeled along with the culture progression and is filled by several new areas, homogeneously distributed, where more ordinate collagen deposition (stained in red) engulfing the cells is observed (see zoomed areas in Figure $\mathbf{7 c}, \mathbf{f}$ ).

The expression of COL2A1 and COL3A1 monitored by an IF assay after 16 days of 3D culture is reported in Figure 8a. The analysis of the scaffolds confirmed the presence of collagen II, stained in green and uniformly distributed along the scaffold nearby the cells' nuclei stained in blue, supporting the hypothesis of newly synthetized matrix. Over the culture time, the area surrounding the cells is indeed progressively filled by type II collagen with more intense and uniform signal.

Gene expression profiles for chondrogenic markers are illustrated inFigure 8b ; scaffolds with $h$ BM-MSCs just after preparation were used as control (time zero). As previously done for the monolayer cultures, gene expression of Sox9 , Col2a1 andAcan positive markers were monitored together with Col1a1 and Col3a1 negative markers by RT-qPCR. In the 3D case, cells displayed progressive and higher upregulation of Col2a1 
up to 5 -fold at Day 16, with respect to simple monolayer culture. Collagen type II level is in agreement with the histology observation, and supported the hypothesis of chondrogenic commitment by the cooperative effect of growth factor and dynamic culture. Conversely, collagen types I and III, markers typically found in fibrocartilageous tissue, were downregulated as it should be expected (Goldring et al., 2006).

Despite a clear upregulation of $S o x 9$ was observed along the monolayer culture, the same overexpression was not observed when cells were organized into 3D construct; this behavior is in agreement with some authors that described unsignificant expression of $\operatorname{Sox} 9$ in 3D culture. These authors justified the observed behavior considering that $\operatorname{Sox} 9$ is an early marker of chondrogenesis and regulates the expression of downstream targets such as COL2A1; however, since it is an early markers for chondrogenesis may has been up regulated earlier and then returned to basal level by day 8 and 16 (Akiyama et al., 2002, Tamaddon et al, 2017). These data are in agreement also with Buxton et al. (2011), who reported that Sox 9 expression was increased in hMSCs at time points shorter than the first week of culture and, then, returned to control levels. Alternatively, within the 3D culture, the exclusive down regulation of Sox9 may not be correlate with Col2a1 expression; indeed, collagen II expression is activated by the coordinated action of the three transcription factors $L$-Sox 5 ,Sox6 , and Sox9 (the so called Sox trio) (Li et al., 2011). Another hypothesis suggested by Kawato et al. (2012) is that the upregulation of Col2a1 may happen with Sox9 -independent mechanism. (Kawato et al., 2012). Acan has been previously described as a late chondrogenic marker; however, as also observed along the 2D culture, its expression appeared downregulated in our 3D setting. Notably, chondrocytes 3D culture have been reported not to express this marker (Jiménez et al., 2019) .q-IF in Figure 8c confirmed a statistical significant increase of collagen II protein staining of 0.4 fold at 16 days; conversely, collagen type III protein staining was progressively reduced at all the time points analyzed; this last observation is particularly interesting because collagen type III is basically overexpressed by undifferentiated $h$ BM-MSC (Ciardulli and Marino et al., 2020) and, therefore, it provided a further suggestion of cells commitment versus a specific phenotype. The fairly good chondrogenic early commitment achieved within the 3D model, again without supplementing dexamethasone, is also in agreement with Buxton et al., (2011) that suggested the use of 3D hydrogels environment to encourage chondrogenesis of hMSCs, avoiding the use of further corticosteroids in the culture medium.

\section{CONCLUSION AND PERSPECTIVES}

The 3D environment hosting PLGA-MCs for hTGFb1 controlled release coupled with the enhanced mass exchange of perfusion culture was effective in inducing chondrogenic commitment in $h$ BM-MSCs. The innovative 3D system described can be designed to perform the sustained release of multiple growth factors and/or other biochemical signals to further explore new solutions for in vitro cartilage repair and regeneration. Indeed, using different MCs loaded with different biological signals for a more complex spatial-temporal delivery, several effects can be achieved. In this sense, the described 3D microenvironment opens concrete perspectives to develop 3D bioengineered models to understand specific molecular and cellular composition of damaged systems.

On the other hand, looking at the clinical applications, the collagen hydrogel microenvironment showed relatively low dynamic viscosity (Knapp et al., 1997) and enriched with PLGA MCs, opens perspective for its future use as an intra-articular injectable advanced therapy for drugs-controlled delivery and/or factors for resident SCs stimulation after its in vivo administration.

\section{ACNOWLEDGMENTS}

The authors acknowledge: MiUR within the framework of PON-RI 2014/2020. Action I.1-"Innovative PhDs with industrial characterization" Cycle XXXIV (D.D. 1090 del 04.05.2018 additional PhD fellowships). PhD project: "Development of a 3D scaffold for local growth factor delivery to improve cartilage tissue engineering". The authors acknowledge NOVAGENIT Srl (www.novagenit.com) for its participation to the $\mathrm{PhD}$ industrial doctorate project.

\section{FUNDING}


This research was developed within the Research contract between the University of Salerno and the IRCCS "Galeazzi", Milan (IT) entitled "Design and implementation of an experimental 3D bioengineered construct for the regeneration of cartilage systems". Scientific Advisors Proff. N. Maffulli and G. Della Porta, Year 2019-20.

\author{
ABBREVIATION LIST \\ $\eta \mathbf{T} \boldsymbol{\Gamma} \boldsymbol{\Phi} \beta 1$ human transforming growth factor b 1 \\ PLGA-MCs poly-lactic-co-glycolic acid microcarriers \\ hBM-MSCs human Bone Marrow Mesenchymal Stem Cells \\ hASC human adipose stem cells \\ COL1A1 type I collagen \\ COL2A1 type II collagen \\ COL3A1 type III collagen \\ SOX9 SRY-Box transcription factor SOX9 \\ ACAN aggrecan \\ RT-qPCR reverse transcription quantitative polymerase chain reaction \\ q-IF semi-quantitative immunofluorescence
}

\title{
REFERENCES
}

Akiyama H, Chaboissier M-C, Martin JF, Schedl A, de Crombrugghe B. 2002. The transcription factor Sox9 has essential roles in successive steps of the chondrocyte differentiation pathway and is required for expression of Sox5 and Sox6. Genes Dev. $16: 2813-2828$.

Alves da Silva ML, Martins A, Costa-Pinto AR, Correlo VM, Sol P, Bhattacharya M, Faria S, Reis RL, Neves NM. 2011. Chondrogenic differentiation of human bone marrow mesenchymal stem cells in chitosan-based scaffolds using a flow-perfusion bioreactor. J. Tissue Eng. Regen. Med. 5 :722-732.

Badylak S, Gilbert T, Myers-Irvin J. 2008. The extracellular matrix as a biologic scaffold for tissue engineering. In: Tissue Eng.Elsevier, pp. 121-143..

Barboni B, Curini V, Russo V, Mauro A, Di Giacinto O, Marchisio M, Alfonsi M, Mattioli M. 2012. Indirect Co-Culture with Tendons or Tenocytes Can Program Amniotic Epithelial Cells towards Stepwise Tenogenic Differentiation. Ed. Wei-Chun Chin. PLoS ONE7 :e30974.

Bustin SA, Benes V, Garson JA, Hellemans J, Huggett J, Kubista M, Mueller R, Nolan T, Pfaff MW, Shipley GL, Vandesompele J, Wittwer CT. 2009. The MIQE Guidelines: Minimum Information for Publication of Quantitative Real-Time PCR Experiments. Clin. Chem.55 :611-622.

Buxton AN, Bahney CS, Yoo JU, Johnstone B. 2011. Temporal Exposure to Chondrogenic Factors Modulates Human Mesenchymal Stem Cell Chondrogenesis in Hydrogels. Tissue Eng. Part A17:371-380.

Ciardulli MC, Marino L, Lovecchio J, Giordano E, Forsyth NR, Selleri C, Maffulli N, Della Porta G. 2020. Tendon and Cytokine Marker Expression by Human Bone Marrow Mesenchymal Stem Cells in a Hyaluronate/Poly-Lactic-Co-Glycolic Acid (PLGA)/Fibrin Three-Dimensional (3D) Scaffold. Cells 9 :1268.

Ciardulli MC, Marino L, Lamparelli EP, Guida M, Forsyth NR, Selleri C, Maffulli N, Della Porta G 2020.A study of Tendon Markers expression promoted by Growth Differentiation Factor-5 on Human-Mesenchymal Stem Cells isolated from Bone Marrow and Umbilical Cord International Journal Molecular Sci., in press . 
Cipollaro L, Ciardulli MC, Porta GD, Peretti GM, Maffulli N. 2019. Biomechanical issues of tissue-engineered constructs for articular cartilage regeneration: in vitro and in vivo approaches. British Medical Bulletin 132 :53-80.

Dehne T, Schenk R, Perka C, Morawietz L, Pruss A, Sittinger M, Kaps C, Ringe J. 2010. Gene expression profiling of primary human articular chondrocytes in high-density micromasses reveals patterns of recovery, maintenance, re- and dedifferentiation. Gene $462: 8-17$.

Della Porta G, Falco N, Reverchon E. 2011. Continuous supercritical emulsions extraction: A new technology for biopolymer microparticles production. Biotechnol. Bioeng. 108 :676-686.

Della Porta G, Ciardulli MC, Maffulli N. 2018. Microcapsule Technology for Controlled Growth Factor Release in Musculoskeletal Tissue Engineering: Sports Med. Arthrosc. Rev. 26 :e2-e9.

Della Porta G, Falco N, Giordano E, Reverchon E. 2013. PLGA microspheres by Supercritical Emulsion Extraction: a study on insulin release in myoblast culture. J. Biomater. Sci. Polym. Ed.24 :1831-1847.

Della Porta, Porta GD, Campardelli R, Reverchon E, Fisher JP. 2015. Synergistic effect of sustained release of growth factors and dynamic culture on osteoblastic differentiation of mesenchymal stem cells: Sustained Growth Factor Release for Osteoblastic Differentiation.J. Biomed. Mater. Res. A 103 :2161-2171.

Della Porta, Reverchon E, Maffulli N. 2017. Biomaterials and Supercritical Fluid Technologies: Which Perspectives to Fabricate Artificial Extracellular Matrix? Curr. Pharm. Des. 23.

El-Sherbiny IM, Yacoub MH. 2013. Hydrogel scaffolds for tissue engineering: Progress and challenges. Glob. Cardiol. Sci. Pract.2013:38.

Falco N, Reverchon E, Della Porta G. 2013. Injectable PLGA/hydrocortisone formulation produced by continuous supercritical emulsion extraction. Int. J. Pharm. 441 :589-597.

Faust HJ, Guo Q, Elisseeff JH. 2019. Cartilage Tissue Engineering. In:.Princ. Regen. Med. Elsevier, pp. 937-952.

Fuentes-Mera L, Camacho A, Moncada-Saucedo NK, Peña-Martínez V. 2017. Current Applications of Mesenchymal Stem Cells for Cartilage Tissue Engineering. In: Pham, PV, editor. Mesenchymal Stem Cells Isol. Charact. Appl. InTech. Eds.

Gimenez-Rota C, Palazzo I, Scognamiglio MR, Mainar A, Reverchon E, Della Porta G. 2019. $\beta$-Carotene, $\alpha$-tocoferol and rosmarinic acid encapsulated within PLA/PLGA microcarriers by supercritical emulsion extraction: Encapsulation efficiency, drugs shelf-life and antioxidant activity.J. Supercrit. Fluids 146 :199207.

Giordano R, Canesi M, Isalberti M, Isaias I, Montemurro T, Viganò M, Montelatici E, Boldrin V, Benti R, Cortelezzi A, Fracchiolla N, Lazzari L, Pezzoli G. 2014. Autologous mesenchymal stem cell therapy for progressive supranuclear palsy: translation into a phase I controlled, randomized clinical study. J. Transl. Med. $12: 14$.

Goldring MB, Tsuchimochi K, Ijiri K. 2006. The control of chondrogenesis. J. Cell. Biochem. 97:33-44.

Govoni M, Berardi AC, Muscari C, Campardelli R, Bonafè F, Guarnieri C, Reverchon E, Giordano E, Maffulli N, Della Porta G. 2017. An Engineered Multiphase Three-Dimensional Microenvironment to Ensure the Controlled Delivery of Cyclic Strain and Human Growth Differentiation Factor 5 for the Tenogenic Commitment of Human Bone Marrow Mesenchymal Stem Cells. Tissue Eng. Part A 23 :811-822.

Govoni M, Lamparelli EP, Ciardulli MC, Santoro A, Oliviero A, Palazzo I, Reverchon E, Vivarelli L, Maso A, Storni E, Donati ME, Ruspaggiari G, Maffulli N, Fini M, Della Porta G, Dallari D. 2020. Demineralized bone matrix paste formulated with biomimetic PLGA microcarriers for the vancomycin hydrochloride controlled delivery: Release profile, citotoxicity and efficacy against S. aureus. Int J Pharm582 :119322. 
Harrell CR, Markovic BS, Fellabaum C, Arsenijevic A, Volarevic V. 2019. Mesenchymal stem cell-based therapy of osteoarthritis: Current knowledge and future perspectives. Biomed. Pharmacother.109:23182326 .

Hellemans J, Mortier G, De Paepe A, Speleman F, Vandesompele J. 2007. qBase relative quantification framework and software for management and automated analysis of real-time quantitative PCR data. Genome Biol. 8 :R19.

Huang JI, Zuk PA, Jones NF, Zhu M, Lorenz HP, Hedrick MH, Benhaim P. 2004. Chondrogenic potential of multipotential cells from human adipose tissue. Plast. Reconstr. Surg. 113 :585-594.

James R, Kumbar SG, Laurencin CT, Balian G, Chhabra AB. 2011. Tendon tissue engineering: adiposederived stem cell and GDF-5 mediated regeneration using electrospun matrix systems. Biomed. Mater.6 :025011.

Jiang X, Huang X, Jiang T, Zheng L, Zhao J, Zhang X. 2018. The role ofSox9 in collagen hydrogel-mediated chondrogenic differentiation of adult mesenchymal stem cells (MSCs). Biomater. Sci.6 :1556-1568.

Jiménez G, Venkateswaran S, López-Ruiz E, Perán M, Pernagallo S, Díaz-Monchón JJ, Canadas RF, Antich C, Oliveira JM, Callanan A, Walllace R, Reis RL, Montañez E, Carrillo E, Bradley M, Marchal JA. 2019. A soft 3D polyacrylate hydrogel recapitulates the cartilage niche and allows growth-factor free tissue engineering of human articular cartilage.Acta Biomaterialia 90 :146-156.

Kawato Y, Hirao M, Ebina K, Shi K, Hashimoto J, Honjo Y, Yoshikawa H, Myoui A. 2012. Nkx3.2 promotes primary chondrogenic differentiation by upregulating Col2a1 transcription. PLoS ONE 7 :e34703.

Knapp DM, Barocas VH, Moon AG, Yoo K, Petzold LR, Tranquillo RT. 1997. Rheology of reconstituted type I collagen gel in confined compression. Journal of Rheology 41 :971-993.

Li H, Haudenschild DR, Posey KL, Hecht JT, Di Cesare PE, Yik JHN. 2011. Comparative analysis with collagen type II distinguishes cartilage oligomeric matrix protein as a primary TGF $\beta$-responsive gene. Osteoarthritis Cartilage 19 :1246-1253.

Li H, Tan C, Li L. 2018. Review of 3D printable hydrogels and constructs. Mater. Des. 159 :20-38.

Li X, Liang Y, Xu X, Xiong J, Ouyang K, Duan L, Wang D. 2019. Cell-to-Cell Culture Inhibits Dedifferentiation of Chondrocytes and Induces Differentiation of Human Umbilical Cord-Derived Mesenchymal Stem Cells. BioMed Res. Int. 2019 :1-11.

Liu Y, Suen C-W, Zhang J, Li G. 2017. Current concepts on tenogenic differentiation and clinical applications. J. Orthop. Transl.9:28-42.

Lovecchio J, Pannella M, Giardino L, Calzà L, Giordano E. 2020. A dynamic culture platform enhances the efficiency of the 3D HUVEC based tube formation assay. Biotechnol. Bioeng. J .117:789-797.

Madry H, Rey-Rico A, Venkatesan JK, Johnstone B, Cucchiarini M. 2014. Transforming Growth Factor Beta-Releasing Scaffolds for Cartilage Tissue Engineering. Tissue Eng. Part B Rev. 20 :106-125.

Mollon B, Kandel R, Chahal J, Theodoropoulos J. 2013. The clinical status of cartilage tissue regeneration in humans. Osteoarthritis Cartilage 21 :1824-1833.

Mueller MB, Tuan RS. 2008. Functional characterization of hypertrophy in chondrogenesis of human mesenchymal stem cells. Arthritis Rheum.58:1377-1388.

Nihant N, Schugens C, Grandfils C, Jerome R, Teyssie P. 1995. Polylactide Microparticles Prepared by Double Emulsion-Evaporation.J. Colloid Interface Sci. 173 :55-65.

Palazzo I, Lamparelli EP, Ciardulli MC, Scala P, Maffulli N, Reverchon E, Santoro A, Della Porta G 2020. PLA-PLGA carrier for Growth Factors delivery fabricated by dense- $\mathrm{CO}_{2}$ : micro/nano-size tailoring, release profiles and cytotoxicity study. J. Supercrit. Fluids, in press. 
Parenteau-Bareil R, Gauvin R, Berthod F. 2010. Collagen-Based Biomaterials for Tissue Engineering Applications. Materials $3: 1863-1887$.

Pasini A, Lovecchio J, Ferretti G, Giordano E. 2019. Medium Perfusion Flow Improves Osteogenic Commitment of Human Stromal Cells. Stem Cells Int. 2019 :1-10.

Pastides P, Chimutengwende-Gordon M, Maffulli N, Khan W. 2013. Stem cell therapy for human cartilage defects: a systematic review.Osteoarthritis Cartilage 21 :646-654.

Puetzer JL, Petitte JN, Loboa EG. 2010. Comparative Review of Growth Factors for Induction of ThreeDimensional In VitroChondrogenesis in Human Mesenchymal Stem Cells Isolated from Bone Marrow and Adipose Tissue. Tissue Eng. Part B Rev. 16 :435-444.

Reyes R, Delgado A, Sánchez E, Fernández A, Hernández A, Evora C. 2014. Repair of an osteochondral defect by sustained delivery of BMP-2 or TGF $\beta 1$ from a bilayered alginate-PLGA scaffold: Osteochondral repair with BMP-2 or TGFß1. J. Tissue Eng. Regen. Med., 8: 521-533.

Rönn K, Reischl N, Gautier E, Jacobi M. 2011. Current Surgical Treatment of Knee Osteoarthritis. Arthritis $2011: 1-9$.

Samavedi S, Poindexter LK, Van Dyke M, Goldstein AS. 2014. Synthetic Biomaterials for Regenerative Medicine Applications. In: . Regen. Med. Appl. Organ Transplant. Elsevier, pp. 81-99..

Shintani N, Hunziker EB. 2011. Differential effects of dexamethasone on the chondrogenesis of mesenchymal stromal cells: influence of microenvironment, tissue origin and growth factor. Eur. Cell. Mater. 22 :302-320..

Siepmann J, Siepmann F. 2008. Mathematical modeling of drug delivery.International Journal of Pharmaceutics $364: 328-343$.

Sophia Fox AJ, Bedi A, Rodeo SA. 2009. The Basic Science of Articular Cartilage: Structure, Composition, and Function. Sports Health Multidiscip. Approach 1 :461-468.

Suchorska WM, Lach MS, Richter M, Kaczmarczyk J, Trzeciak T. 2016. Bioimaging: An Useful Tool to Monitor Differentiation of Human Embryonic Stem Cells into Chondrocytes. Ann. Biomed. Eng.44 :18451859 .

Tamaddon M, Burrows M, Ferreira SA, Dazzi F, Apperley JF, Bradshaw A, Brand DD, Czernuszka J, Gentleman E. 2017. Monomeric, porous type II collagen scaffolds promote chondrogenic differentiation of human bone marrow mesenchymal stem cells in vitro. Sci Rep 7 :43519.

Tirado DF, Palazzo I, Scognamiglio M, Calvo L, Della Porta G, Reverchon E. 2019. Astaxanthin encapsulation in ethyl cellulose carriers by continuous supercritical emulsions extraction: A study on particle size, encapsulation efficiency, release profile and antioxidant activity.J. Supercrit. Fluids 150 :128-136.

Tığlı RS, Cannizaro C, Gümüşderelioğlu M, Kaplan DL. 2011. Chondrogenesis in perfusion bioreactors using porous silk scaffolds and hESC-derived MSCs. J. Biomed. Mater. Res. A 96A :21-28.

Trucillo E, Bisceglia B, Valdrè G, Giordano E, Reverchon E, Maffulli N, Della Porta G. 2019. Growth factor sustained delivery from poly-lactic-co-glycolic acid microcarriers and its mass transfer modeling by finite element in a dynamic and static three-dimensional environment bioengineered with stem cells. Biotechnol. Bioeng.116 :1777-1794.

de Winter JCF. 2013. Using the Student's t-test with extremely small sample sizes. Pract. Assess. Res. Eval. 18 .

Xu J, Wang W, Ludeman M, Cheng K, Hayami T, Lotz JC, Kapila S. 2008. Chondrogenic Differentiation of Human Mesenchymal Stem Cells in Three-Dimensional Alginate Gels. Tissue Eng. Part A14:667-680.

Yeatts AB, Fisher JP. 2011. Tubular Perfusion System for the Long-Term Dynamic Culture of Human Mesenchymal Stem Cells. Tissue Eng. Part C Methods 17 :337-348. 
Yoo JU, Barthel TS, Nishimura K, Solchaga L, Caplan AI, Goldberg VM, Johnstone B. 1998. The chondrogenic potential of human bone-marrow-derived mesenchymal progenitor cells. J. Bone Joint Surg. Am. 80 :1745-1757.

Zhang W, Ouyang H, Dass CR, Xu J. 2016. Current research on pharmacologic and regenerative therapies for osteoarthritis. Bone Res. $4: 15040$.

Zhang Y, Jordan JM. 2010. Epidemiology of Osteoarthritis. Clin. Geriatr. Med. 26 :355-369.

Zhong L, Huang X, Karperien M, Post J. 2016. Correlation between Gene Expression and Osteoarthritis Progression in Human. Int. J. Mol. Sci. 17 :1126.

\section{FIGURE CAPTIONS}

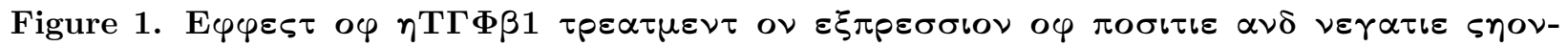

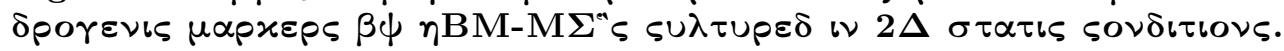

hBM-MSCs were treated with two different concentrations of hTGFß1 (1 ng/mL and $10 \mathrm{ng} / \mathrm{mL})$ over 16 days. Untreated cells for all the time-points were used as control. At the lowest concentration of hTGF $\beta 1$ of $1 \mathrm{ng} / \mathrm{mL}$, Sox 9 levels are more than doubled after 16 days of treatment (a ); concentration of hTGF $\beta 1$ of $10 \mathrm{ng} / \mathrm{mL}$ induces a fourfold $S o x 9$ up-regulation and the doubling of Col2a1 expression at day 16 (b ).

${ }^{* *} p<0.01,{ }^{* * *} p<0.001$ and ${ }^{* * * *} p<0.0001$ vs. control (two-way ANOVA, $\mathrm{n}=3$ ).

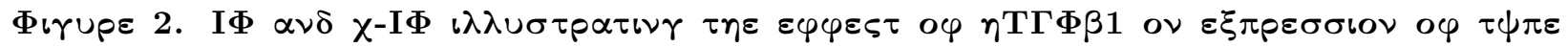

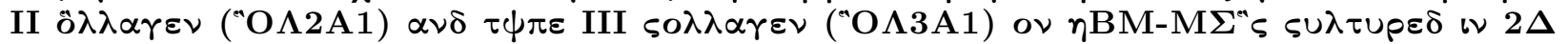

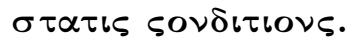

hBM-MSCs were treated with two different concentrations of hTGF $\beta 1$ ( 1 and $10 \mathrm{ng} / \mathrm{mL})$ for 16 days. hBMMSCs showed a slight basal expression of positive COL2A1 marker, which increased after 8 and 16 days of culture mainly when treated with $10 \mathrm{ng} / \mathrm{mL}$ of hTGF $\beta 1$. The spontaneous upregulation of negative COL3A1 marker at $16 \mathrm{~d}$ was significantly reduced upon treatment with $10 \mathrm{ng} / \mathrm{mL}$ hTGF $\beta 1$. When $1 \mathrm{ng} / \mathrm{mL}$ was supplemented, hBM-MSCs aggregated into a spindle shaped system (a) see arrowhead at day 16; no spindle aggregates were monitored when a concentration of $10 \mathrm{ng} / \mathrm{mL}$ was used $\backslash$ sout.

${ }^{*} p<0.05,{ }^{* *} p<0.01,{ }^{* * *} p<0.001$ and ${ }^{* * * *} p<0.0001$, one-way ANOVA). $\mathrm{n}=3$

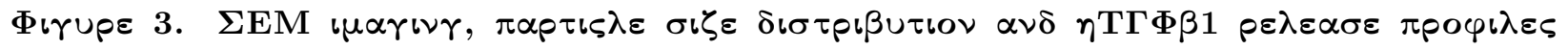

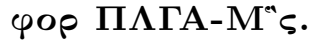

SEM imaging (a) and particle size distribution (b) of PLGA carriers obtained after SEE process; size distribution data are expressed as volume percentage. hTGF $\beta 1$ release profiles monitored at $37^{\circ} \mathrm{C}$ using an ELISA-based assay expressed as percentage; $\mathrm{n}=3$ (c) ; hTGF $\beta 1$ amount (ng/mL/day) released within each 3D-scaffold loaded with $6.6 \mathrm{mg}$ of carriers (d).

Figure 4. Schematic illustration of extrusion and gelification process (a), image of 3D scaffold system (b), confocal image of carrier and cells organization within 3D scaffold (c), FE-SE imaging of collagen fiber morphology $(d)$.

Schematic illustration of extrusion and gelification process of the functionalized scaffold (a). Image of 3D scaffold within the multiwell perfusion system after 16 days of culture (b). Confocal imaging of the scaffold system loaded with $h$ BM-MSCs (DAPI nuclei highlighted in blue) and PLGA-MCs loaded with Rhodamine $\mathrm{B}$ (highlighted in red): a uniform carrier distribution is evident within 3D collagen scaffold (c). The internal structure of collagen scaffold fibers is observed by scanning electron microscope after scaffold freeze-drying (d).

Figure 5. Live/dead assay to check cell viability during scaffold assembly protocol. 
Confocal microscopy imaging of 3D scaffold system (a) with live/dead signal semiquantitative evaluation (b) of hBM-MSCs viability right after collagen extrusion and cross-linking. Semiquantitative evaluation indicated an excellent cell viability (2.9\% of dead cells).

Figure 6. Perfusion bioreactor and FEM analysis of dynamic environment conditions.

Schematic representation of dynamic environment where the $3 \mathrm{D}$ scaffolds were cultivated; $1 \mathrm{~mL} / \mathrm{min}$ was pumped continuously along the 16 days of culture (a); perfusion bioreactor overall system pictures and scaffold details (b) ; FEM analysis of nutrient consumption $(\mathbf{c}, \mathbf{f})$ and waste production $(\mathbf{d}, \mathbf{g})$ in static $(\mathbf{c}, \mathbf{d})$ vs. dynamic $(\mathbf{f}, \mathbf{g})$ condition, concentration are represented as mean value and expressed as $\mathrm{mol} / \mathrm{m}^{3}$; FEM analysis of medium velocity profile (grey lines) and distribution (horizontal cross-section) within a single culture well (e), the perfusion flow is oriented along the y direction.

Figure 7. Hematoxylin \& Eosin and Sirius red staining of $3 \mathrm{D}$ bioengineered system at different time-points.

Both staining conditions showed a scaffold matrix that was progressively remodeled by cells with large areas in where new extracellular collagen matrix was deposited; this effect is more evident at Day 16. Sirius red staining highlighted the collagen original 3D scaffold matrix formed by random network of red stained fibers at Day zero; then, along the culture the collagen matrix showed the presence of large areas still stained in red, but organized with more aligned matrix. Cells nuclei are evident within these new area, as highlighted by zoomed areas.

Figure 8. q-IF analyses of type II and type III collagens and RT-qPCR gene expression profiles for positive and negative chondrogenic markers in $3 \mathrm{D}$ culture.

IF showed the progressive collagen type II deposition in the areas surrounding the cells; type III collagen protein expression was not detected in all the time points analyzed (a). mRNA levels of different positive and negative chondrogenic markers (Col1a1,Col2a1, Col3a1, Sox9 and Acan ) were monitored using a RTqPCR; untreated $h$ BM-MSCs were used as control. Col2a1 mRNA levels showed a stronger up-regulation (4.9-fold) at day 16; (b) ${ }^{*} p<0.05,{ }^{* *} p<0.01,{ }^{* * *} p<0.001$ and ${ }^{* * * *} p<0.0001$ (two-way ANOVA, $\mathrm{n}$ $=3)$. q-IF confirmed the trend indicating statistical significant increase of collagen type II signal at Day 16 (stained in green) and significant reduction of collagen III signal (stained in red) (c) . Scale bar: 50um. ${ }^{*} p$ $<0.05,{ }^{* *} p<0.01,{ }^{* * *} p<0.001$ and ${ }^{* * * *} p<0.0001$ (one-way ANOVA, $\mathrm{n}=3$ ).

(a)

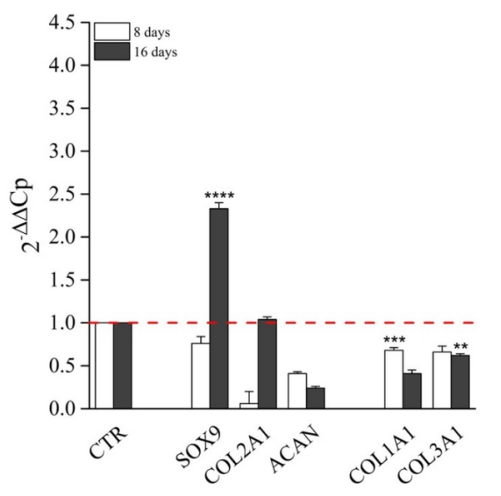

(b)

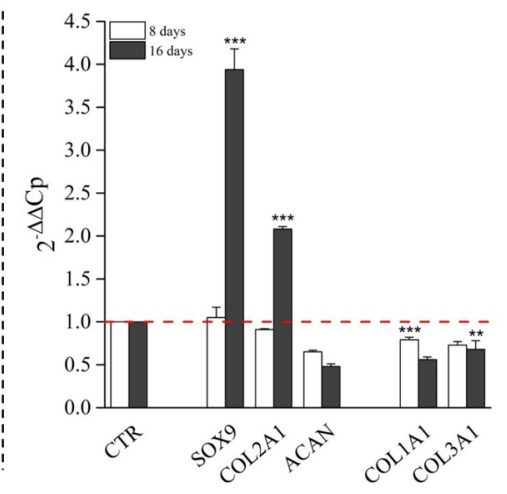

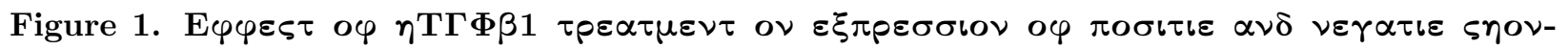

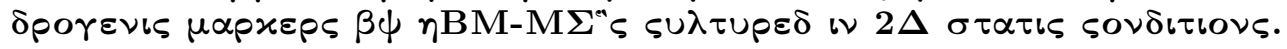


hBM-MSCs were treated with two different concentrations of hTGF $\beta 1(1 \mathrm{ng} / \mathrm{mL}$ and $10 \mathrm{ng} / \mathrm{mL})$ over 16 days. Untreated cells for all the time-points were used as control. At the lowest concentration of hTGF $\beta 1$ of $1 \mathrm{ng} / \mathrm{mL}$, Sox 9 levels are more than doubled after 16 days of treatment (a ); concentration of hTGF $\beta 1$ of $10 \mathrm{ng} / \mathrm{mL}$ induces a fourfold Sox9 up-regulation and the doubling of Col2a1 expression at day 16 (b ).

${ }^{*} p<0.05,{ }^{* *} p<0.01,{ }^{* *} p<0.001$ and ${ }^{* * * *} p<0.0001$ vs. control (two-way ANOVA, $\mathrm{n}=3$ ).

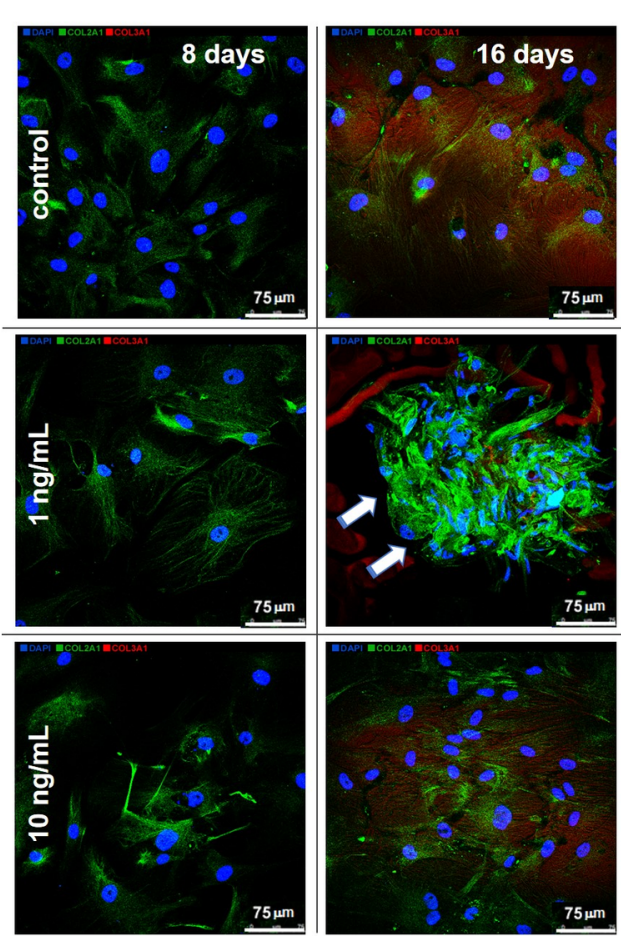

(a)

(b)
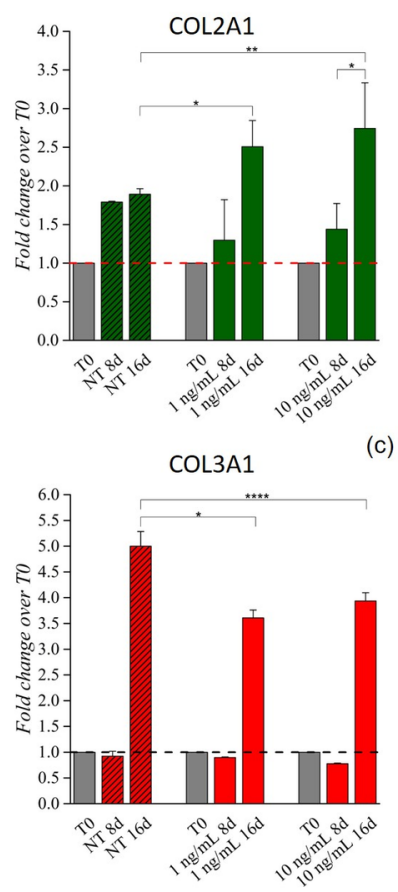

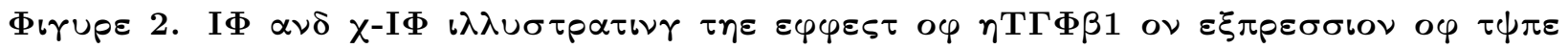

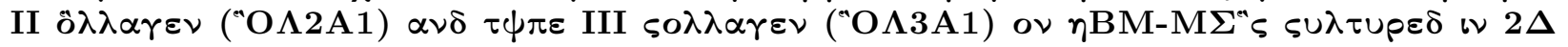

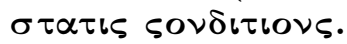

hBM-MSCs were treated with two different concentrations of hTGF $\beta 1$ ( 1 and $10 \mathrm{ng} / \mathrm{mL}$ ) for 16 days. hBMMSCs showed a slight basal expression of positive COL2A1 marker, which increased after 8 and 16 days of culture mainly when treated with $10 \mathrm{ng} / \mathrm{mL}$ of hTGF $\beta 1$. The spontaneous upregulation of negative COL3A1 marker at $16 \mathrm{~d}$ was significantly reduced upon treatment with $10 \mathrm{ng} / \mathrm{mL} \mathrm{hTGF} \beta 1$. When $1 \mathrm{ng} / \mathrm{mL}$ was supplemented, hBM-MSCs aggregated into a spindle shaped system (a) see arrowhead at day 16; no spindle aggregates were monitored when a concentration of $10 \mathrm{ng} / \mathrm{mL}$ was used $\backslash$ sout.

${ }^{*} p<0.05,{ }^{* *} p<0.01,{ }^{* * *} p<0.001$ and ${ }^{* * * *} p<0.0001$, one-way ANOVA). $\mathrm{n}=3$ 

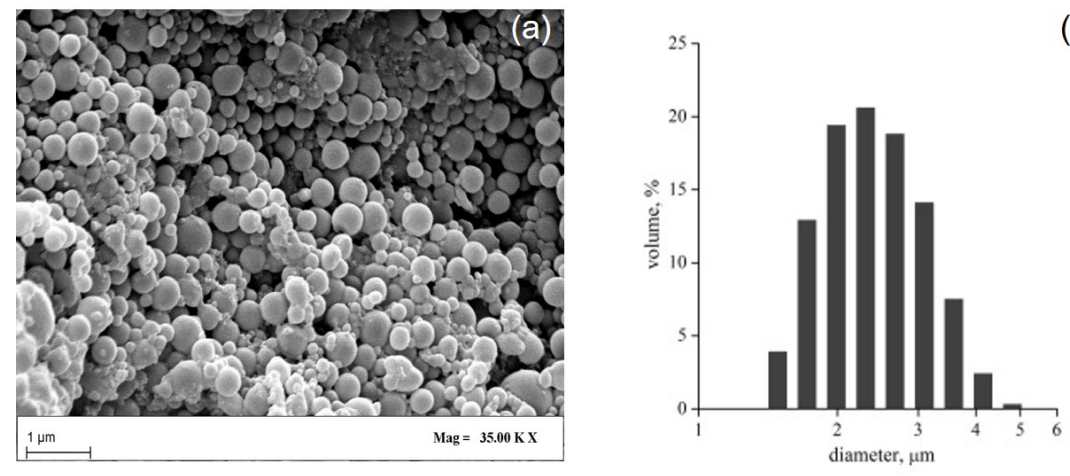

(b)

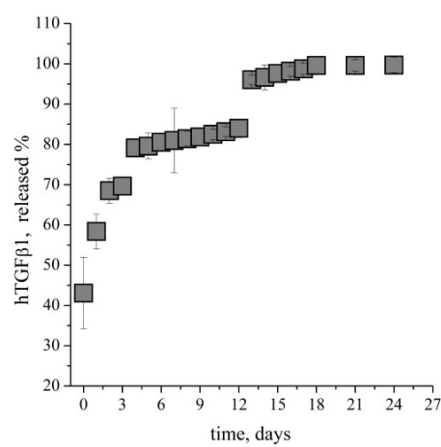

(c)

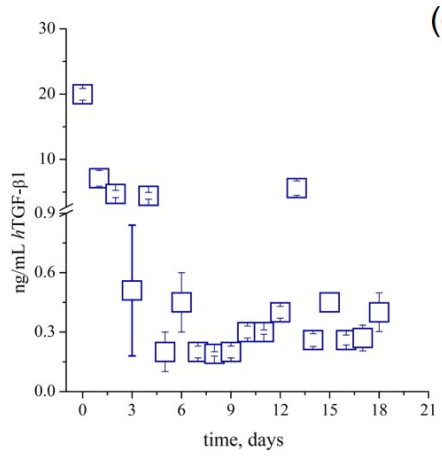

(d)

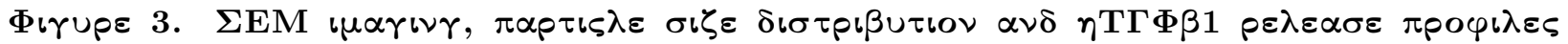

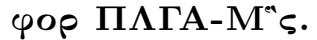

SEM imaging (a) and particle size distribution (b) of PLGA carriers obtained after SEE process; size distribution data are expressed as volume percentage. hTGF $\beta 1$ release profiles monitored at $37^{\circ} \mathrm{C}$ using an ELISA-based assay expressed as percentage; $\mathrm{n}=3$ (c) ; hTGF $\beta 1$ amount $(\mathrm{ng} / \mathrm{mL} /$ day $)$ released within each 3D-scaffold loaded with $6.6 \mathrm{mg}$ of carriers (d). 

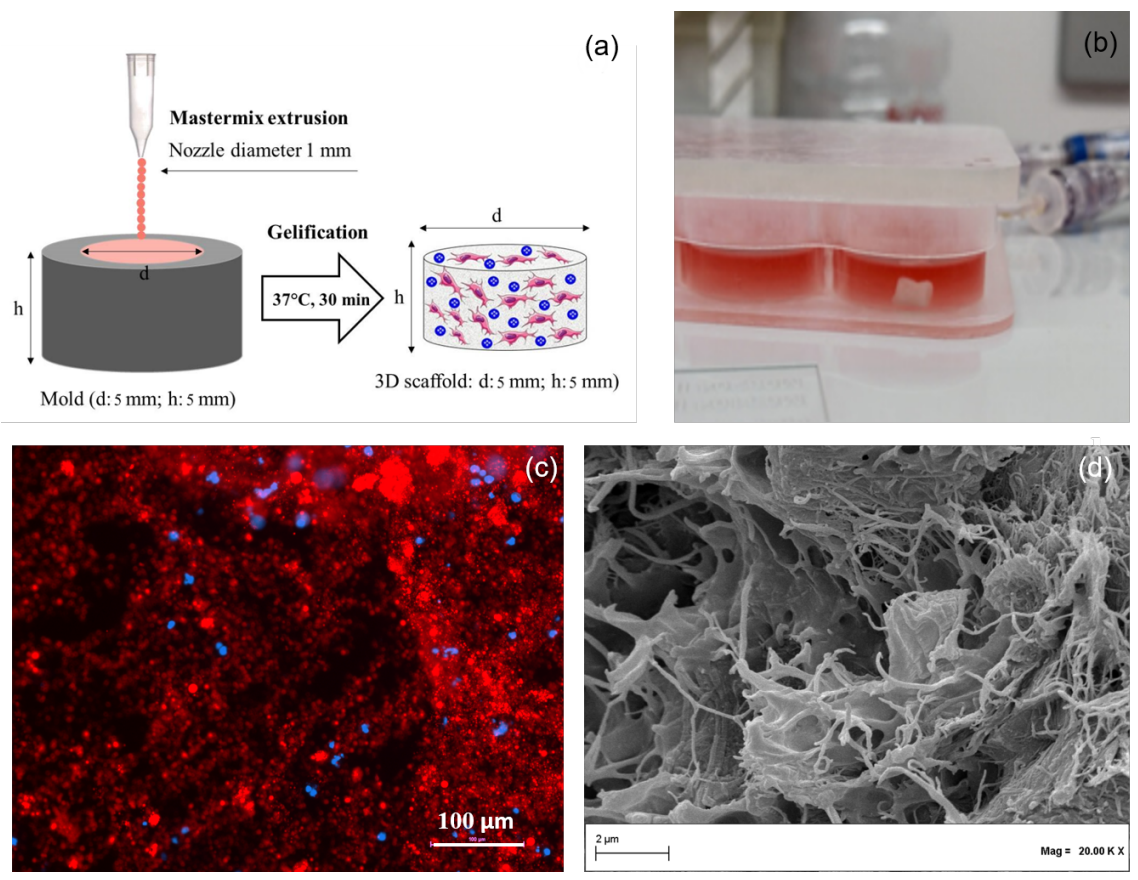

Figure 4. Schematic illustration of extrusion and gelification process (a), image of 3D scaffold system (b), confocal image of carrier and cells organization withn 3D scaffold (c), FE-SE imaging of collagen fiber morphology $(d)$.

Schematic illustration of extrusion and gelification process of the functionalized scaffold (a). Image of 3D scaffold within the multiwell perfusion system after 16 days of culture (b). Confocal imaging of the scaffold system loaded with $h$ BM-MSCs (DAPI nuclei highlighted in blue) and PLGA-MCs loaded with Rhodamine $\mathrm{B}$ (highlighted in red): a uniform carrier distribution is evident within 3D collagen scaffold (c). The internal structure of collagen scaffold fibers is observed by scanning electron microscope after scaffold freeze-drying (d). 


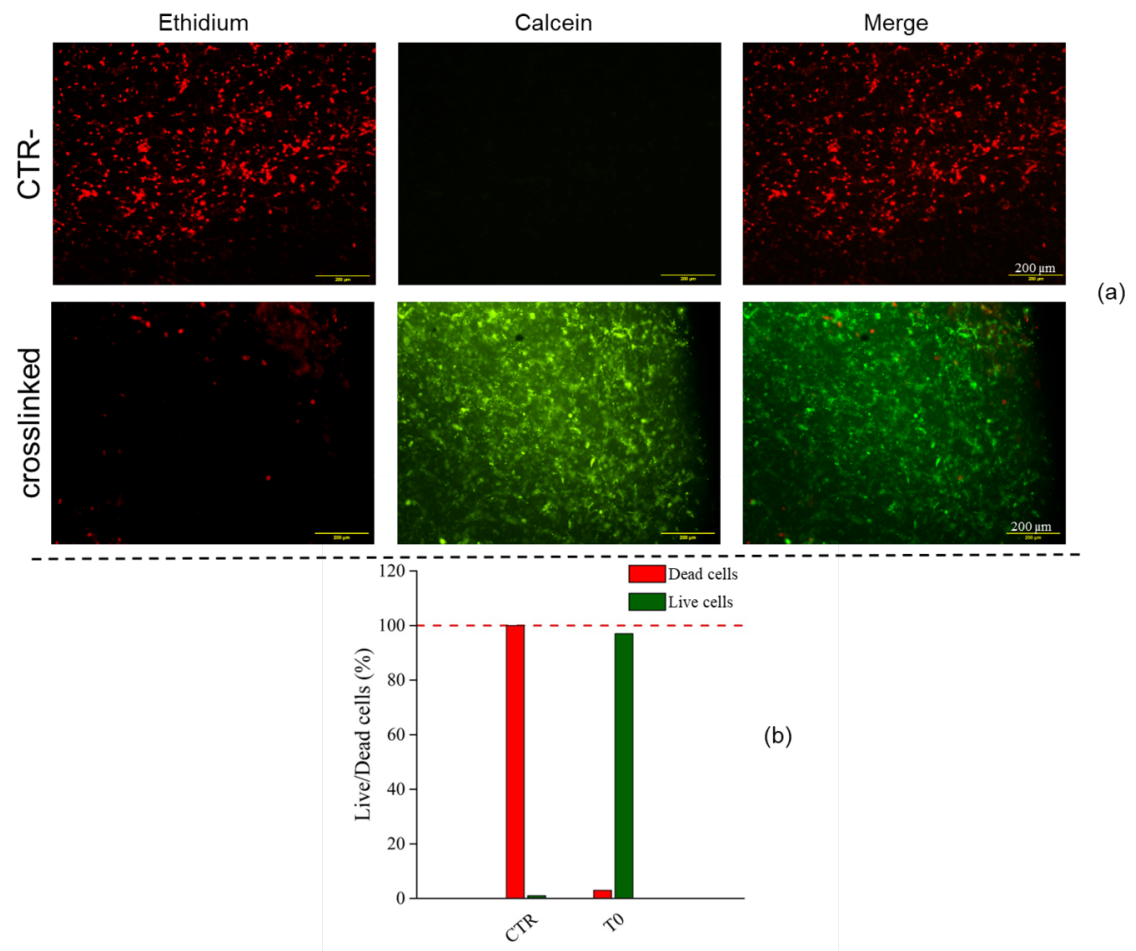

Figure 5. Live/dead assay to check cell viability during scaffold assembly protocol.

Confocal microscopy imaging of 3D scaffold system (a) with live/dead signal semiquantitative evaluation (b) of hBM-MSCs viability right after collagen extrusion and cross-linking. Semiquantitative evaluation indicated an excellent cell viability (2.96\% of dead cells).

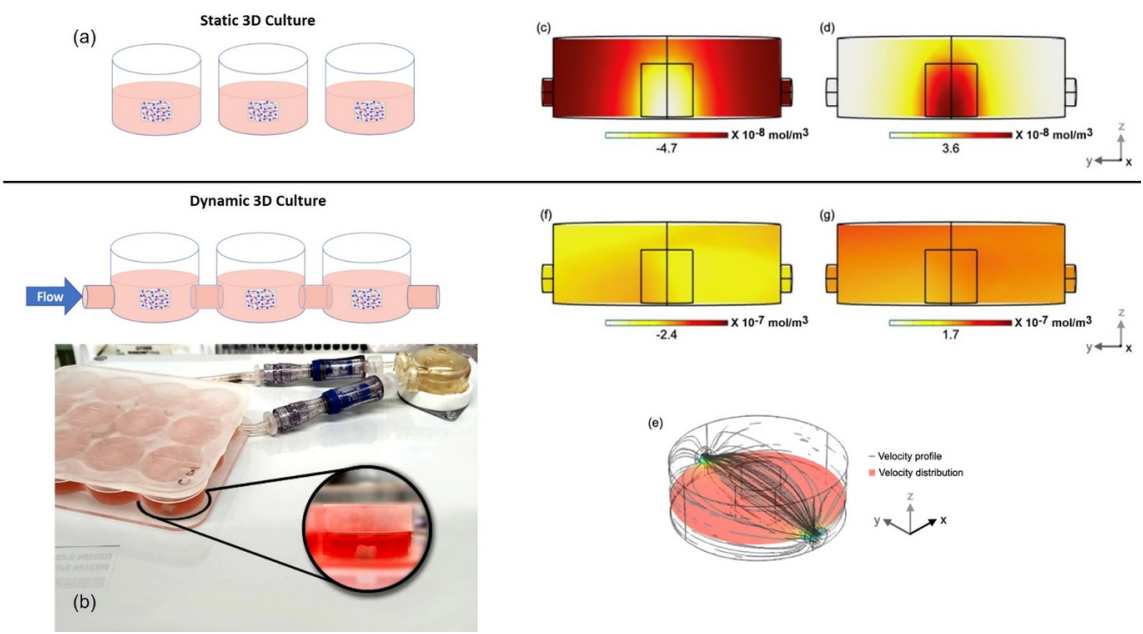

Figure 6. Perfusion bioreactor and FEM analysis of dynamic environment conditions.

Schematic representation of dynamic environment where the $3 \mathrm{D}$ scaffolds were cultivated; $1 \mathrm{~mL} / \mathrm{min}$ was pumped continuously along the 16 days of culture (a); perfusion bioreactor overall system pictures and 
scaffold details (b) ; FEM analysis of nutrient consumption $(\mathbf{c}, \mathbf{f})$ and waste production $(\mathbf{d}, \mathbf{g})$ in static(c,d) vs. dynamic (f,g) condition, concentration are represented as mean value and expressed as $\mathrm{mol} / \mathrm{m}^{3}$; FEM analysis of medium velocity profile (grey lines) and distribution (horizontal cross-section) within a single culture well (e), the perfusion flow is oriented along the y direction.
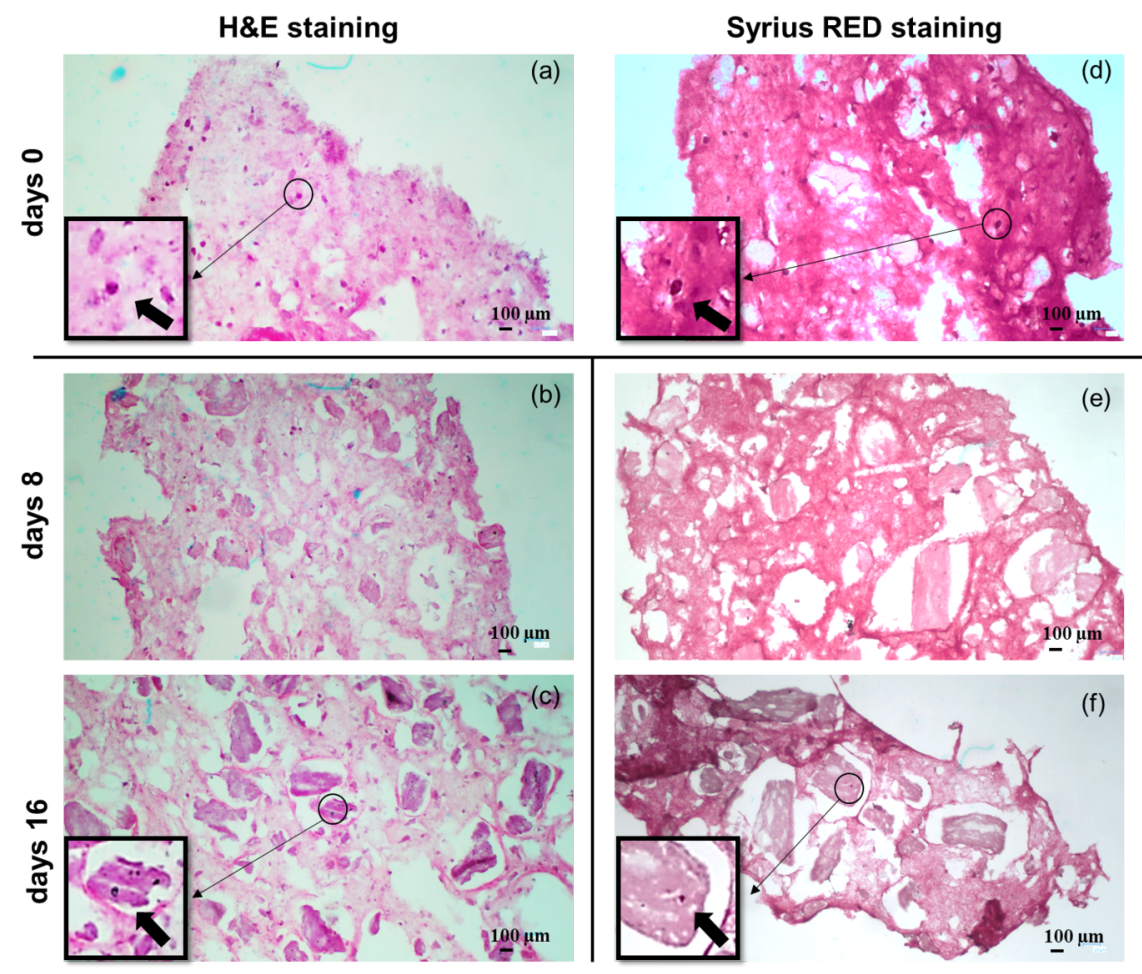

Figure 7. Hematoxylin \& Eosin and Sirius red staining of 3D bioengineered system at different time-points.

Both staining conditions showed a scaffold matrix that was progressively remodeled by cells with large areas in where new extracellular collagen matrix was deposited; this effect is more evident at Day 16. Sirius red staining highlighted the collagen original 3D scaffold matrix formed by random network of red stained fibers at Day zero; then, along the culture the collagen matrix showed the presence of large areas still stained in red, but organized with more aligned matrix. Cells nuclei are evident within these new area, as highlighted by zoomed areas. 
(a)

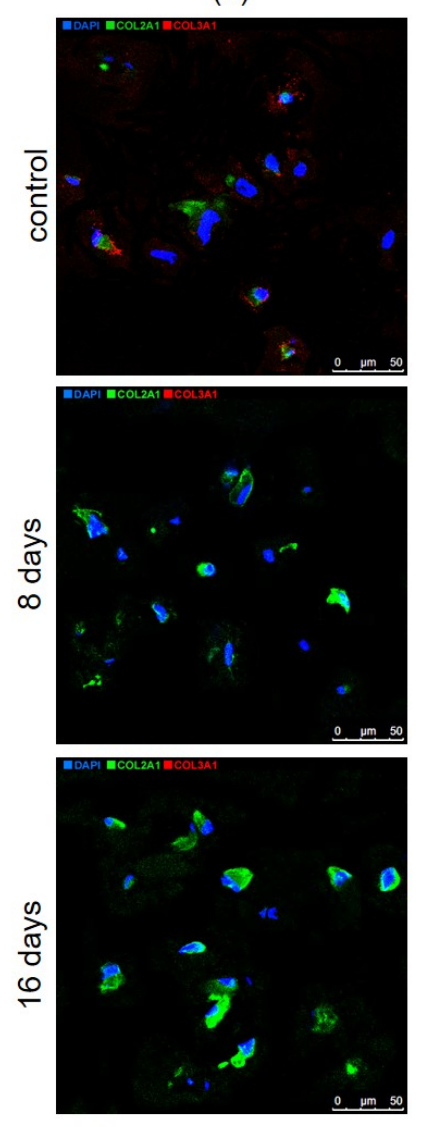

(b)

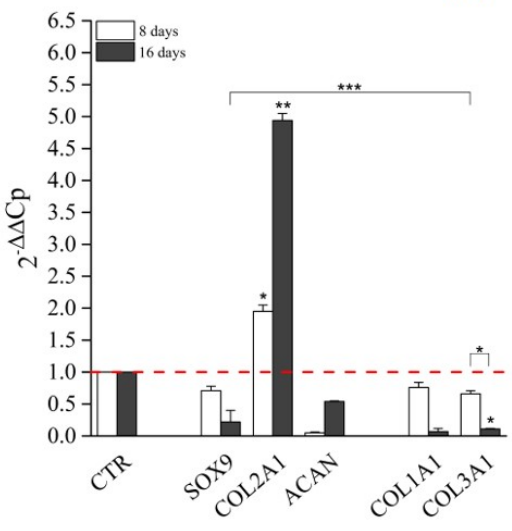

(c)

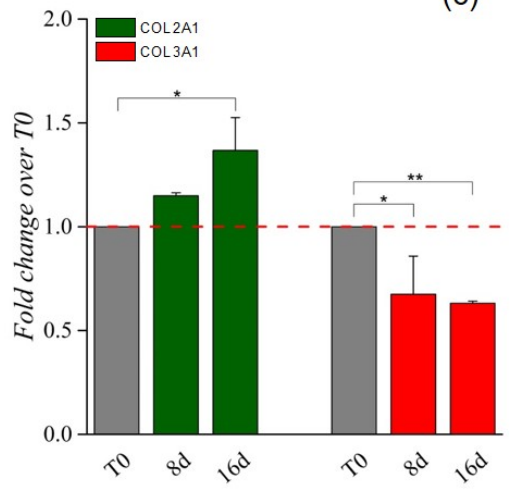

Figure 8. q-IF analyses of type II and type III collagens and RT-qPCR gene expression profiles for positive and negative chondrogenic markers in $3 \mathrm{D}$ culture.

IF showed the progressive collagen type II deposition in the areas surrounding the cells; type III collagen protein expression was not detected in all the time points analyzed (a). mRNA levels of different positive and negative chondrogenic markers (Col1a1,Col2a1, Col3a1, Sox9 and Acan ) were monitored using a RTqPCR; untreated $h$ BM-MSCs were used as control. Col2a1 mRNA levels showed a stronger up-regulation (4.9-fold) at day 16 ; (b) ${ }^{*} p<0.05,{ }^{* *} p<0.01,{ }^{* * *} p<0.001$ and ${ }^{* * * *} p<0.0001$ (two-way ANOVA, $\mathrm{n}$ $=3$ ). q-IF confirmed the trend indicating statistical significant increase of collagen type II signal at Day 16 (stained in green) and significant reduction of collagen III signal (stained in red) (c) . Scale bar: 50um. ${ }^{*} p$ $<0.05,{ }^{* *} p<0.01,{ }^{* * *} p<0.001$ and ${ }^{* * * *} p<0.0001$ (one-way ANOVA, $\mathrm{n}=3$ ). 